\title{
The psychological impact of participating in colorectal cancer screening by faecal immuno-chemical testing - the Australian experience
}

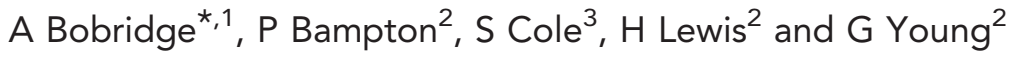 \\ ${ }^{1}$ School of Nursing \& Midwifery, University of South Australia, Room 44, Level 6, Centenary Building, North Terrace, Adelaide, \\ South Australia 5000, Australia; ${ }^{2}$ Flinders Centre for Innovation in Cancer Flinders University, Bedford Park, South Australia 5042, \\ Australia and ${ }^{3}$ Bowel Health Service Repatriation General Hospital, Daws Road, Daws Park, South Australia 5041, Australia
}

Background: Occult blood-based colorectal cancer (CRC) screening may result in adverse psychological outcomes for participants. The aims of this study were to measure the psychological consequences of participating in screening at key points along the screening and diagnostic pathway, and examine variation over time within or between test outcome groups.

Methods: A total of 301 people (positives $=165$, negatives $=136$ ) aged 50-76 years were surveyed via validated psychological questionnaires after result notification, post colonoscopy (positives only) and 1 year following result notification.

Results: Negatives scored significantly higher in quality of life domains and lower state anxiety, anger and depression in comparison to positives both after result notification and at 1 year follow-up. Positives had significantly decreased state anxiety and depression at 1 year and improvement in HLoC power and reduced screening decision doubtfulness post colonoscopy. Positives experienced heightened CRC risk perception both after result notification and at 1 year follow-up in comparison to negatives, but reported less difficulty participating in ongoing screening.

Conclusions: In positives, increased anxiety and doubtfulness about the decision to screen declined over time. Lower CRC risk perception in negatives indicates the need for education to promote CRC screening participation.

Worldwide, colorectal cancer (CRC) is the third most common cancer in men and the second in women (WHO, 2010). To reduce the burden of CRC, various population screening programmes have been introduced (European Commission, 2010; Hoff and Dominitz, 2010; Ross, 2010) aimed at reducing morbidity and mortality through early detection. One of these programs - faecal immuno-chemical testing (FIT) can be achieved entirely through the postal system, with participants receiving a positive or negative result letter with follow-up advice.

However, studies have shown that those who test positive in such screening programmes experience elevated anxiety levels (Parker et al, 2002; Kapidzic et al, 2012). Possible distress may also arise from receiving a false positive result, undergoing a follow-up colonoscopy or from anticipating an unfavourable diagnosis (Bowel Cancer Screening Pilot Monitoring and Evaluation Steering Committee, 2005; Wackerbarth et al, 2008).

Although the anxiety elicited from CRC screening is well established, less is known about the impact of this type of screening on quality of life (QOL), self-efficacy, cancer risk perception and screening decision making, all of which have been shown to influence future screening intentions and uptake (McQueen et al, 2008; Lewis et al, 2010; Dillard et al, 2012). Therefore, this prospective cohort study aimed to determine the short- and longterm psychological consequences of receiving a positive or negative result in FIT-based CRC screening programmes in Australia at key points along the screening and diagnostic pathway. 


\section{MATERIALS AND METHODS}

Colorectal cancer population screening in Australia. Population screening was introduced in 2008 through the National Bowel Cancer Screening Program (NBCSP). The NBCSP Register sends eligible people a pre-invitation letter followed by an invitation pack, which includes an immunochemical test kit for detection of faecal occult blood. Participants collect two faecal samples, which are mailed to the programme pathology laboratory for analysis. Test results are sent to the participant and their nominated general practitioner, and positive participants are advised to undergo diagnostic colonoscopy. Approximately $6 \%$ of participants who undergo this form of screening test positive (Bowel Cancer Screening Pilot Monitoring and Evaluation Steering Committee, 2005).

Study eligibility criteria. Study participants had to be aged 50-74 years when they completed CRC screening. The positive group had a positive FIT following a NBCSP screening test and had been referred to metropolitan public hospitals for diagnostic colonoscopy. The negative group had a negative FIT through a public hospital co-ordinated population screening research programme, which followed exactly the same screening process as the NBCSP.

Ethics approval for this study was granted by the Southern Adelaide Clinical Human Research Ethics Committee.

Study design. Potential participants were mailed a study invitation, six questionnaires, a participant information sheet, an 'optout' form and a reply paid envelope. Completion and return of the questionnaires indicated consent. Those who did not opt out or reply were sent a second and third reminder after 4 and 6 weeks of the initial mail-out (Dillman et al,1993; Edwards et al, 2002).

Survey schedule. FIT positives were sent a set of questionnaires (1) after positive FIT result notification (ARN) but prior to undergoing colonoscopy; (2) after undergoing colonoscopy; and (3) at 12 months after the original result notification. FIT negatives were sent a set of questionnaires (1) after negative FIT result notification and (2) at 12 months after the original result notification. The average time from ARN to the first questionnaire being posted was 1-3 months, with the post colonoscopy questionnaire (for positive participants) sent 1-3 weeks after the procedure.

\section{Questionnaires}

1. Demographic Information Survey: age, gender, postcode (for Index of Relative Socioeconomic Disadvantage (IRSAD), Australian Bureau of Statistics, 2013), place of birth, first language, employment status and education level.

2. Quality of Life - Short-Form 36: evaluates physical functioning, physical and emotional role limitations, vitality, mental health, social functioning, body pain, general health and health transition (Sharples et al, 2000; Basnov et al, 2009). Answers are weighted out of 100. Lower scores indicate poorer outcomes.

3. Anxiety \& Depression - Spielberger State-Trait Inventory: measures anxiety, curiosity, anger and depression traits as well as the participant's current state with respect to these indices (Spielberger, 2010). The higher the number, the worse the indice.

4. Multi-Dimensional Health Locus of Control: includes domains of 'internal control' and 'power' (measuring belief in personal control over health) and 'chance', 'doctor' and 'other people' (representing belief that other people or things control one's health) (Wallston et al, 1978). A higher aggregated score indicates stronger agreement with each domain.

5. Decision Evaluation Scale applied to CRC screening: uses a 5-point Likert scale to evaluate the participant's level of satisfaction with their decision to undertake CRC screening
(Stalmeier et al, 2005). A higher score equals stronger agreement with the item.

6. Colorectal Cancer Risk: uses a 7-point Likert scale to evaluate the participant's perception of CRC risk (risk perception), confidence in participating in screening (self-efficacy) and response to screening (response efficacy) (Levy et al, 2006). A higher number equals higher risk perception and efficacy.

Analysis. The sample size was calculated assuming 95\% probability with $80 \%$ power to detect shifts in key outcome scores of $20 \%$. All data were analysed using SPSS v19. As one objective of this study was to identify significant differences in outcomes between groups, analyses were conducted using paired and independent-sample $t$-tests. Chi-square analysis was performed on frequency data. Multivariate regression analyses were undertaken to identify psychosocial constructs jointly associated with test outcomes while controlling for differences in demographic factors between groups.

\section{RESULTS}

In all 520 questionnaires were sent $A R N$ (positives $=305$, negatives $=215$ ), with 301 questionnaires returned (positives $=165$, negatives $=136$; response rate $=58 \%$; see Figure 1 ).

Sample characteristics. The mean age of negatives was greater than that of positives, at 61.8 vs 59.5years $(P=0.009)$. The positive group had $43 \%$ females and $57 \%$ males, while the negative group had $53 \%$ females and $47 \%$ males $(P=N S)$. The place of birth for the majority of participants was Australia (69\% of positives, $76 \%$ of negatives) followed by the United Kingdom (23\% of positives, $14 \%$ of negatives). Similar patterns in employment status and education qualifications were observed between groups. There was a significant difference between groups for the IRSAD, with more positives living in low IRSAD suburbs (45\% vs $30 \% ; P=0.034$ ). The few participants with English as second-language were in the positive group $(P=0.039)$. Multivariate regression analysis showed that differences in sample characteristics did not significantly influence study outcomes.

Quality of life. Positives showed no significant changes in QOL domains post colonoscopy or at 1 year relative to ARN. At both $\mathrm{ARN}$ and at 1 year, negatives demonstrated greater physical functioning $(P=0.016$ and 0.017 , respectively), vitality $(P=0.015$ and 0.008$)$, mental health $(P=0.026$ and 0.049$)$, social functioning $(P=0.036$ and 0.004$)$, general health $(P=0.025$ and 0.036$)$ and less role limitation related to emotional health problems $(P=0.012$ and 0.020) compared to positives (Table 1).

Anxiety and depression. Positives experienced a significant improvement in the trait depression post colonoscopy $(P=0.023)$ and improvements in state anxiety and depression at 1 year $(P=0.050$ and 0.041 , respectively), while negatives had a significant improvement in the trait depression $(P=0.000)$ at 1 year. ARN and 1-year comparison demonstrated significantly higher (worse) state anxiety $(P=0.000$ and 0.014$)$, anger $(P=0.005$ and 0.004$)$ and depression $(P=0.011$ and 0.000$)$ in positives compared to negatives (Table 1$)$.

Health locus of control. Post colonoscopy, positives demonstrated a significant improvement in the power domain $(P=0.042)$, indicating more self-control over their health. At 1 year, positives felt their health was less reliant on doctors (significant decrease in the doctor domain $(P=0.033))$. There were no significant changes in the negative group or between groups ARN or at 1 year (Table 1 ). 


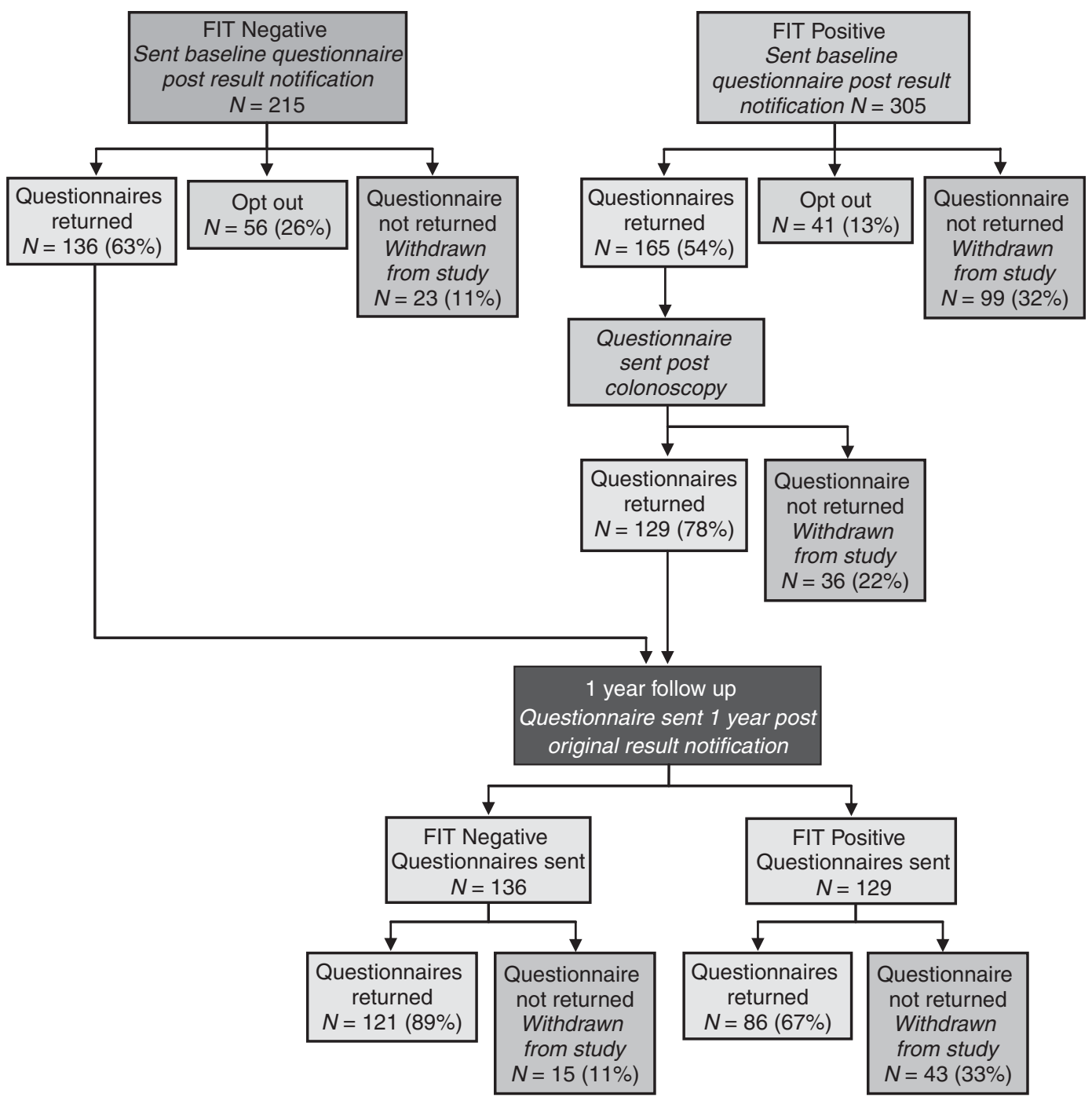

Figure 1. Returned questionnaires, study withdrawals and opt-out numbers.

Decision evaluation. ARN, positives had greater doubtfulness about their screening decision $(P=0.045)$ and felt less satisfied about the information they received $(P=0.036)$ compared to negatives. Positives experienced a significant decrease in doubt related to their CRC screening decision post colonoscopy $(P=0.004)$ and a decrease in wanting information about screening options at 1 year $(P=0.022)$, while negatives experienced a significant decrease in satisfaction with information received $(P=0.012$; Table 1$)$.

Colorectal cancer risk. Post colonoscopy, positives reported greater likelihood of developing CRC if they did not participate in screening $(P=0.005)$, and increased seriousness of suffering from $\operatorname{CRC}(P=0.022)$, but less difficulty in participating in ongoing CRC screening $(P=0.012)$. Positives also experienced a significant improvement in self-efficacy post colonoscopy $(P=0.025)$, which decreased slightly at 1 year $(P=0.012)$. Negatives experienced no significant changes in cancer risk perception or self-efficacy at 1 year. ARN and 1-year comparisons demonstrated that negatives rated their likelihood of developing CRC, if they did not participate in screening $(P=0.002$ and 0.000$)$ and in comparison to the average person $(P=0.002$ and 0.039$)$, as lower relative to positives (Table 1).

\section{DISCUSSION}

We believe that this study is one of the most comprehensive assessments of the psychological impacts of FIT CRC screening, demonstrating that while a positive result does cause an element of anxiety, this improves over time. However, anxiety does remain higher than negatives, most likely associated with an increased perception of the risk of developing CRC. Undergoing screening is also associated with an increase in the autonomy of decision making and recognition that surveillance does reduce CRC risk, suggesting that overall there is perhaps a psychological benefit for FIT-positive participants.

Undergoing colonoscopy did not significantly change the QOL domains for positives, which is consistent with the QOL screening studies undertaken by Niv et al (2012) and Pizzo et al (2011). This is in contrast to a study by Taupin et al (2006) demonstrating statistically significant improvements in mental health, role limitations related to emotions and vitality post colonoscopy in FITpositive participants. Together these studies strongly indicate that undergoing CRC screening is not detrimental to the screenee's QOL.

State anxiety, anger and depression were consistently lower in negatives compared to positives, although state anxiety did decrease in FIT positives post colonoscopy and at 1 year. 


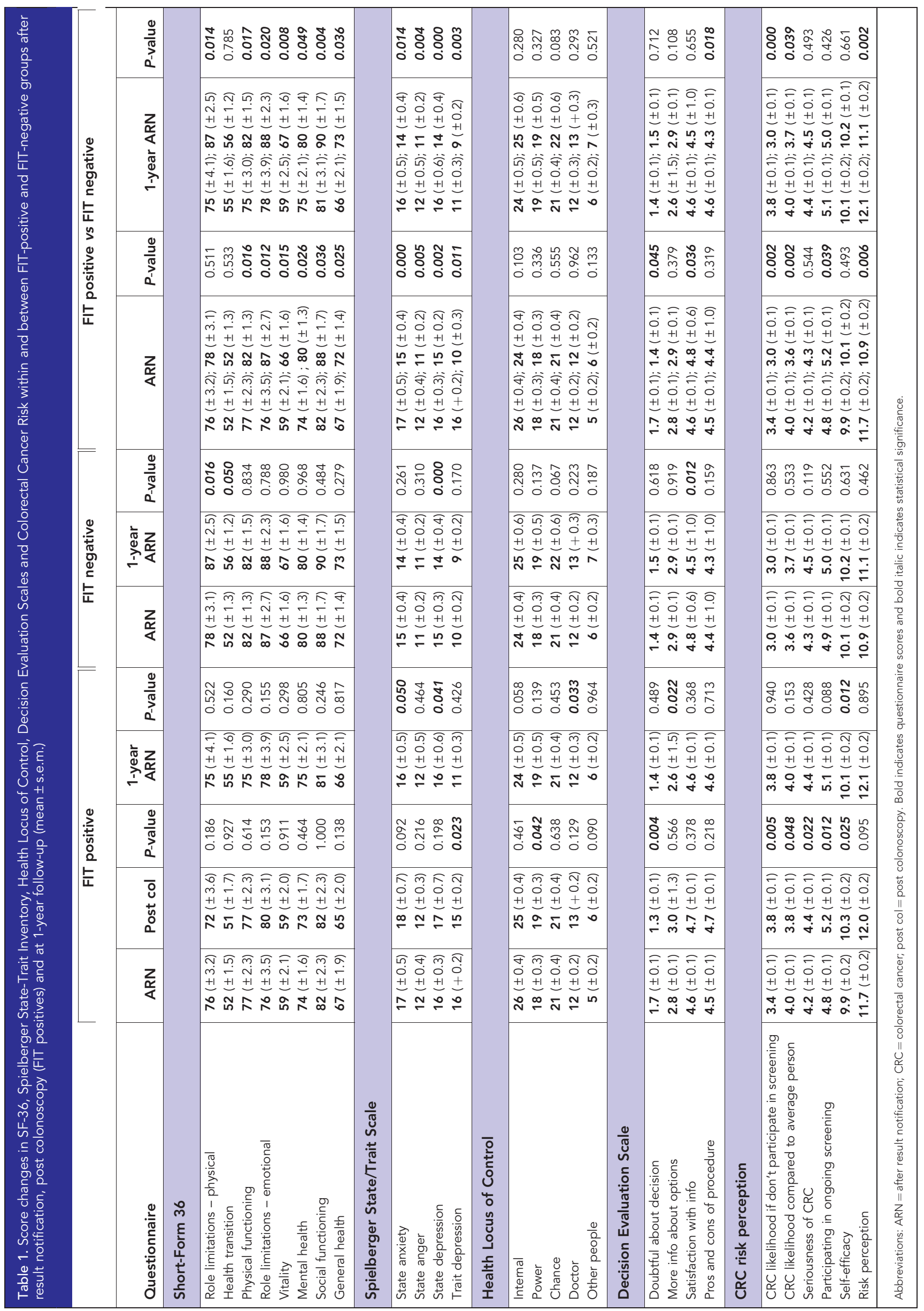


The reduction in state anxiety within the positive group is consistent with other studies by Brasso et al (2010), Parker et al (2002) and a more recent study that included measurements pre FIT and also demonstrated significantly higher mean scores and mood disturbance in FIT positives compared to negatives, with a drop in these same parameters 4 months post testing (Laing et al, 2014).

FIT positives experienced a significant improvement in the HLoC power domain post colonoscopy, indicating that undergoing such a procedure is perhaps an empowering experience in terms of perceived control over one's health. Indeed, a sense of self-empowerment and self-efficacy has been demonstrated to positively influence cancer screening behaviour, in terms of decision making, intentions and uptake (Consedine et al, 2007; McQueen et al, 2008; Luszczynska et al, 2012).

Positives showed significantly higher doubt in relation to their screening decision. This decreased following colonoscopy, probably associated with removal of apprehension about diagnostic outcome. Positives also reported that they would find it less difficult to participate in ongoing CRC screening, with the intention to continue screening after making an initial decision to screen also being supported by Lewis et al (2010).

A contrast was seen in CRC risk perception, with negatives reporting significantly less likelihood of developing CRC in comparison to positives both $\mathrm{ARN}$ and at 1 year. In addition, positives reported an increase in seriousness of CRC after colonoscopy. This demonstrated discrepancy is of importance, as higher perceived CRC susceptibility is a positive predictor of higher screening intentions (Azaiza and Cohen, 2008; Dillard et al, 2012), while a lower perceived susceptibility is a negative indicator (Wackerbarth et al, 2008). This indicates that perhaps negatives require education on their overall CRC risk, with the aim of encouraging participation in future screening.

Limitations. One limitation of this study is the smaller sample size in comparison to other studies. However, outcomes are congruent with other studies in terms of minimal adverse effects on QOL indicators and the transience of anxiety following a positive result.

This study lacks a 'true' baseline (i.e. a measure of outcomes prior to CRC screening). Owing to privacy and confidentiality restrictions, researchers are unable to access the Australian register that stores information and co-ordinates the national CRC screening mail-out. Therefore, there is currently no way of obtaining pre-testing data from participants.

Lastly, the sample comprised predominantly those born in Australian or the United Kingdom and may not represent the impact of CRC screening on different cultural groups. Despite these limitations, the study provides new insights into the psychological impacts of FIT-based CRC screening in Australia.

\section{ACKNOWLEDGEMENTS}

This study was funded by the South Australian Government Strategic Health Research Program.

\section{CONFLICT OF INTEREST}

The authors declare no conflict of interest.

\section{REFERENCES}

Australian Bureau of Statistics (2013) Socio-Economic Indexes for Areas (SEIFA) 2011. ABS Catalogue no. 2033.0.55.001, pp 1-8. Commonwealth of Australia: Canberra.
Azaiza F, Cohen M (2008) Colorectal cancer screening, intentions, and predictors in Jewish and Arab Israelis: a population-based study. Health Educ Behav 35(4): 478-493.

Basnov M, Kongsved SM, Bech P, Hjollund NH (2009) Reliability of short form-36 in an Internet- and a pen-and-paper version. Info Health Soc Care 34(1): 53-58.

Bowel Cancer Screening Pilot Monitoring and Evaluation Steering Committee (2005) A Qualitative Evaluation of Opinions, Attitudes and Behaviours Influencing the Bowel Cancer Screening Pilot Program. Screening Monograph No. 2/2005, p 8. Commonwealth of Australia: Australia.

Bowel Cancer Screening Pilot Monitoring and Evaluation Steering Committee (2005) Australia's Bowel Cancer Screening Pilot and Beyond. Final Evaluation Report. p 67. Commonwealth of Australia: Australia.

Brasso K, Ladelund S, Frederiksen BL, Jorgensen T (2010) Psychological distress following fecal occult blood test in colorectal cancer screening - a population-based study. Scand J Gastroenterol 45(10): 1211-1216.

Consedine NS, Horton D, Magai C, Kukafka R (2007) Breast screening in response to gain, loss, and empowerment framed messages among diverse, low-income women. J Health Care Poor Underserved 18(3): 550-566.

Dillard AJ, Ferrer RA, Ubel PA, Fagerlin A (2012) Risk perception measures' associations with behavior intentions, affect, and cognition following colon cancer screening messages. Health Psych 31(1): 106-113.

Dillman DA, Sinclair MD, Clark JR (1993) Effects of questionnaire length, respondent-friendly design, and a difficult question on response rates for occupant-addressed census mail surveys. Pub Opin Quart 57(3): 289-304.

Edwards P, Roberts I, Clarke M, DiGuiseppi C, Pratap S, Wentz R, Kwan I (2002) Increasing response rates to postal questionnaires: systematic review. BMJ 324(7347): 1183.

European Commission (2010) European Guidelines for Quality Assurance in Colorectal Cancer Screening and Diagnosis, 1st edn. European Union: Luxembourg (Chapter 1).

Hoff G, Dominitz J (2010) Contrasting US and European approaches to colorectal cancer screening: which is best? Gut 59(3): 407-414.

Kapidzic A, Korfage IJ, van Dam L, van Roon AH, Reijerink JC, Zauber AG, van Ballegooijen M, Kuipers EJ, van Leerdam ME (2012) Quality of life in participants of a CRC screening program. Br J Canc 107(8): 1295-1301.

Laing SS, Bogart A, Chubak J, Fuller S, Green BB (2014) Psychological distress after a positive fecal occult blood test result among members of an integrated healthcare delivery system. Canc Epidemiol Biomarkers Prev 23(1): 154-159.

Levy AG, Shea J, Sankey V, Quistberg A, Armstrong K (2006) Measuring perceptions of breast cancer risk. Canc Epidemiol Biomarkers Prev 15: 1893-1898.

Lewis CL, Couper MP, Levin CA, Pignone MP, Zikmund-Fisher BJ (2010) Plans to stop cancer screening tests among adults who recently considered screening. J Gen Intern Med 25(8): 859-864.

Luszczynska A, Durawa AB, Scholz U, Knoll N (2012) Empowerment beliefs and intention to uptake cervical cancer screening: three psychosocial mediating mechanisms. Wom Health 52(2): 162-181.

McQueen A, Tiro JA, Vernon SW (2008) Construct validity and invariance of four factors associated with colorectal cancer screening across gender, race, and prior screening. Canc Epidemiol Biomarkers Prev 17(9): 2231-2237.

Niv Y, Bogolavski I, Ilani S, Avni I, Gal E, Vilkin A, Levi Z (2012) Impact of colonoscopy on quality of life. Eur J Gastroenterol Hepatol 24(7): 781-786.

Parker MA, Robinson MH, Scholefield JH, Hardcastle JD (2002) Psychiatric morbidity and screening for colorectal cancer. J Med Screen 9(1): 7-10.

Pizzo E, Pezzoli A, Stockbrugger R, Bracci E, Vagnoni E, Gullini S (2011) Screenee perception and health-related quality of life in colorectal cancer screening: a review. Value Health 14(1): 152-159.

Ross WA (2010) Colorectal cancer screening in evolution: Japan and the USA. J Gastroenterol Hepatol 25(Suppl 1): S49-S56.

Sharples LD, Todd CJ, Caine N, Tait S (2000) Measurement properties of the Nottingham Health Profile and the Short Form 36 health status measures in a population sample of elderly people living at home: results from ELPHS. Br J Health Psych 5(Part 3): 217-233.

Spielberger CD (2010) State-Trait Anxiety Inventory. Corsini Encyclopedia of Psychology. John Wiley and Sons: New Jersey.

Stalmeier PFM, Roosmalen MS, Verhoef LCG, Hoekstra-Weebers JE, Oosterwijk JC, Moog U, Hoogerbrugge N, van Daal WA (2005) The Decision Evaluation Scales. Pat Educ Counsel 57: 286-293. 
Taupin D, Chambers SL, Corbett M, Shadbolt B (2006) Colonoscopic screening for colorectal cancer improves quality of life measures: a population-based screening study. Health Qual Life Outcomes 4: 82.

Wackerbarth SB, Peters JC, Haist SA (2008) Modelling the decision to undergo colorectal cancer screening: insights on patient preventive decision making. Med Care 46(9 Suppl 1): S17-S22.
Wallston KA, Strudler-Wallston B, DeVellis R (1978) Development of the multidimensional health locus of control (MHLC) scales. Health Educ Monogr 6(2): 160-170.

World Health Organization, International Agency for Research on Cancer (2010) GLOBOCAN 2008. Estimated Cancer Incidence, Mortality, Prevalence and Disability-adjusted life years (DALYs) Worldwide in 2008. Lyon, France. 